\title{
The belief revision model: Asymmetrical effects of noncontingency on human covariation learning
}

\author{
ANTONIO MALDONADO, ANDRÉS CATENA, ANTONIO CÁNDIDO, and INMACULADA GARCÍA \\ University of Granada, Granada, Spain
}

\begin{abstract}
A noncontingent experience affects the subsequent detection of positive and negative contingencies between the same events. Experiments 1 and 2 showed that such preexposure can produce both an impairment in the detection of subsequent positive contingency and a facilitation of a negative one, independent of the level of contingency during the contingent phase. Experiment 3 raised difficulties for a model that assumes that associations to the context can explain this asymmetrical effect. Experiment 4 suggested that the different weights usually assigned to the different types of trials when computing the contingency between events can change as a result of a noncontingent experience with the same events. This change supports an account of the asymmetrical effect by a belief revision model based on a mechanism that updates the weights of the different trial types as a function of previous experience. More generally, the belief revision model is a statistical (i.e., nonassociative) model of learning that is capable of accounting for trial-order effects, which have long posed problems for statistical models.
\end{abstract}

The ability to detect relationships among events in our environment is a major component of adaptive behavior. Recent research has studied such ability, using covariation judgments in humans. The results (Allan, 1993; Shanks, 1995) have usually indicated that such judgments are similarly affected by the same conditions as are conditioned responses in animal learning. The similar effects of contiguity, contingency (Shanks, Pearson, \& Dickinson, 1989), or cue competition in phenomena such as blocking (Chapman \& Robbins, 1990), and predictive validity (Baker, Mercier, Vallée-Tourangeau, Frank, \& Pan, 1993; Price \& Yates, 1995; Wasserman, 1990) have led researchers to propose that human covariation judgments and conditioned response learning probably rely on similar mechanisms (Miller \& Matute, 1996).

Two main classes of mechanisms have been proposed to explain human covariation learning. First, researchers working within the framework of animal learning (e.g., Chapman \& Robbins, 1990; Shanks \& Dickinson, 1987; Wasserman, 1990) have stressed the formal similarity between classical (and instrumental) conditioning and human contingency tasks, and they have claimed that animal learning and covariation detection can be explained by a similar associative mechanism, such as, for example, the

This research was supported by a DGICYT Spanish Grant PB940801 to A.C. and A.M. We gratefully acknowledge the helpful comments of N. MacKintosh, A. Dickinson, D. Shanks, and especially R. Miller. We are also grateful to I. Valderrama for her help with the English version and to J. C. Perales, M. Ruz, M. L. Ramírez, and A. Molina for their help in conducting the last two experiments. We are deeply indebted to R. Miller for his kind final editing of the manuscript. Correspondence should be addressed to A. Maldonado, Departamento de Psicología Experimental, Facultad de Psicología, Campus de Cartuja, Universidad de Granada, 18071 Granada, Spain (e-mail: anmaldo@ platon.ugr.es).
Rescorla and Wagner rule (1972; see Allan,1993; Price \& Yates, 1995; Shanks, López, Darby, \& Dickinson, 1996, for reviews).

Second, another class of models originated in the seminal work of Edwards (1954), who introduced the idea that humans behave as if they calculate probabilities and act as intuitive statisticians. According to these rulebased (statistical) models, humans use a cognitive mechanism to calculate a statistical rule (e.g., $\Delta P$ ) to make their judgments (see, e.g., Cheng \& Novick, 1992; Jenkins \& Ward, 1965; Shaklee \& Mims, 1982; Waldmann \& Holyoak, 1992).

Both types of models can explain many of the human contingency phenomena, and their predictions concerning asymptotic values usually are very similar (Chapman \& Robbins, 1990). In fact, Shanks (1995) has pointed out that perhaps the best way to distinguish between these models may be through the manipulation of trial order (see, also, Chapman, 1991) in sequential learning or processing tasks.

One animal learning phenomenon that directly assesses the effect of previous learning is learned irrelevance. Baker and MacKintosh $(1977,1979)$ showed that previous experience in which two stimuli (a conditioned stimulus [CS] and an unconditioned stimulus [US]) were uncorrelated produced a subsequent impairment of behavior control on either an excitatory or an inhibitory conditioning task with the same CS and US. This learned irrelevance effect can be accommodated, according to rule-based models, by the assumption that humans and animals compute the statistical rule on a trial-by-trial basis that includes both previous experience and subsequent conditioning sessions (Baker et al., 1993; Cheng \& Novick, 1992).

On the other hand, associative models have assumed a context-blocking effect owing to the associative strength acquired by the context during the noncontingent preex- 
posure to the US, in order to explain subsequent learning impairment in excitatory conditioning (Holland \& Rescorla, 1982). However, these associative models cannot easily accommodate the observed delayed acquisition of conditioned inhibition after noncontingent preexposure (Baker \& MacKintosh, 1977, 1979). Moreover, recent evidence has shown that the effects of learned irrelevance on excitatory conditioning cannot be reduced to a context-blocking or a latent inhibition effect, as the serial presentation of the US alone and the CS alone during several sessions did not produce as much delay as did learned irrelevance, in which both stimuli are presented uncorrelated, which is problematic for most associative and statistical models (Bennet, Maldonado, \& MacKintosh, 1995; Matzel, Schachtman, \& Miller, 1988). However, recent research suggests that learned irrelevance might be reduced to the sum of the context specificity of latent inhibition and the US preexposure effects, which raises problems for a unique interpretation of learned irrelevance (Bonardi \& Hall, 1996).

Collectively, the results can be accommodated by neither normative nor associative models, and they suggest that preexposure to uncorrelated cues produces a change in their processing and/or the attention given to these stimuli in the future (Baker \& MacKintosh, 1977), which reduces the associability of a CS for a specific US that is a consequence of the predictive history of that CS for that US (Bennet et al.,1995) and provides evidence of a mechanism that is uniquely sensitive to the lack of correlation between cues prior to conditioning (Matzel et al., 1988).

In human instrumental covariation learning, exposure to previous noncontingency (or uncontrollability) between a response and a consequence usually produces lower judgments than does a prior controllable task or no previous experience, when humans are asked to estimate subsequent positive contingencies between responses and consequences (Maldonado, Martos, \& Ramírez, 1991). But in select circumstances, this treatment leads to accurate judgments or an illusion of control (Alloy \& Tabachnik, 1984; Matute, 1995). According to the learned helplessness model proposed by Maier and Seligman (1976), an expectation of action-outcome independence is established during the uncontrollable treatment and then interferes with the detection of the instrumental contingency in subsequent situations in which control over outcomes was made possible. However, the effect of helplessness on subsequent negative contingencies has never been studied, and the actual results on positive contingencies do not allow the rejection of an associative account based on a context-blocking effect that is similar to the one proposed as an explanation for learned irrelevance.

Therefore, the first objective of this research was to make a systematic study of the effects of a prior experience, in which two events independent of the subjects' responses (i.e., Pavlovian) were uncorrelated, on the ability to detect subsequent positive or negative relationships between the same events and to see if we could obtain a learned irrelevance effect in human covariation learning.
By analogy with Baker and MacKintosh (1977), we expected to see delay as a result of prior exposure in both cases. The task was to estimate the strength of the relationship between two asymmetrical (i.e., with levels defined by presence or absence) dichotomous variablesa fictitious symptom (frontopigmentosis) and a disease (tropical herpiasis) - based upon all the fictitious patients that had been seen up to the moment of the response. We defined four patient (trial) types: The $a$ patient has the symptom and the disease; the $b$ patient has the symptom, but not the disease; the $c$ patient has the disease, without having the symptom; and the $d$ patient does not have the symptom or the disease. This allowed the manipulation of contingency both during a first, uncorrelated phase and during a second, correlated one.

If the subjects were rational and took into account all the patients seen from the beginning of training, normative models of learning would predict that the subjects with previous uncorrelated experience should make less positive and less negative judgments in the detection of subsequent positive and negative contingencies, respectively, than would naive subjects without such previous experience (see Table 1). In accordance with these predictions, in the first two experiments, the effect of previous noncontingent experience on different levels of positive and negative subsequent contingency between the same events was assessed, whereas in the last two experiments, an explanation of the effects observed in Experiments 1 and 2 was sought.

\section{EXPERIMENT 1}

This experiment was designed to study an analogue of the learned irrelevance effect in human covariation learning (Baker \& MacKintosh, 1977; Bennet et al., 1995). The subjects were asked to judge the relationship between a fictitious symptom and a fictitious disease after seeing a given number of patients. In two noncontingent groups, the symptom and the disease were first presented uncorrelated across 32 patients and were then either positively $(+.5)$ or negatively $(-.5)$ correlated. In two control groups, however, the symptom and the disease were always positively $(+.5)$ or negatively $(-.5)$ correlated (see Table 1 ) from the beginning of training. According to previous results in classical conditioning (Baker \& MacKintosh, 1977), it was expected that the noncontingency experience should produce a similar impairment in the subsequent detection of positive and negative covariation, defined as less accurate judgments of the actual positive or negative contingency.

\section{Method}

Subjects. Data were obtained from 64 undergraduate students with normal or corrected vision from the introductory psychology classes at the University of Granada (Spain), and they received course credit for their participation in the study.

Instruments and Stimuli. An Amstrad PC-82386SX computer controlled a high-resolution screen (VGA) for the presentation of stimuli, the sequence of trials, and the collection of responses. The 
Table 1

Design of Experiment 1

\begin{tabular}{|c|c|c|c|c|c|}
\hline \multirow[b]{2}{*}{ Group } & \multicolumn{2}{|c|}{ Phase 1} & \multicolumn{2}{|c|}{ Phase 2} & \multirow[b]{2}{*}{$\begin{array}{c}\text { Total } \\
\text { Contingency }\end{array}$} \\
\hline & $\begin{array}{c}\text { Trial Types } \\
\text { in Each Block }\end{array}$ & $\begin{array}{c}\text { Phase } \\
\text { Contingency }\end{array}$ & $\begin{array}{c}\text { Trial Types } \\
\text { in Each Block }\end{array}$ & $\begin{array}{c}\text { Phase } \\
\text { Contingency }\end{array}$ & \\
\hline $\mathrm{G} 0+5$ & $2 \mathrm{~A}, 2 \mathrm{~B}, 2 \mathrm{C}, 2 \mathrm{D}$ & 0 & $3 \mathrm{~A}, 1 \mathrm{~B}, 1 \mathrm{C}, 3 \mathrm{D}$ & +.5 & +.27 \\
\hline $\mathrm{G}+5$ & & & $3 \mathrm{~A}, 1 \mathrm{~B}, 1 \mathrm{C}, 3 \mathrm{D}$ & +.5 & +.50 \\
\hline $\mathrm{G} 0-5$ & $2 \mathrm{~A}, 2 \mathrm{~B}, 2 \mathrm{C}, 2 \mathrm{D}$ & 0 & $1 \mathrm{~A}, 3 \mathrm{~B}, 3 \mathrm{C}, \mathrm{ID}$ & -.5 & -.27 \\
\hline $\mathrm{G}-5$ & & & $1 \mathrm{~A}, 3 \mathrm{~B}, 3 \mathrm{C}, 1 \mathrm{D}$ & -.5 & -.50 \\
\hline
\end{tabular}

Note-The total number of trials (or patients) during each phase was 32. After each block of 8 patients, a judgment was requested. The number in the groups indicated the contingency between the symptom and the disease $(+5,0$, or -5$)$. A description of the trial types is provided in the text. Total contingency takes into account all the trials from the beginning of training.

stimuli were photographs of different people's faces, randomly selected without replacement from a pool of 68 fictitious patients of both sexes and various ages. Light spotting in orange on the forehead indicated that the patient had the symptom (called frontopigmentosis). A face with large areas of spotting in bright red meant that the patient had the disease (named tropical herpiasis). Faces without colored spots indicated the absence of both the symptom and the disease.

Design. The subjects were randomly assigned to four groups, and their task was to estimate the degree of the relationship between the symptom (frontopigmentosis) and the disease (tropical herpiasis) after having seen a set of patients. We define four patient types (trials): The $a$ patient has the symptom and the disease; the $b$ patient has the symptom alone; the $c$ patient has the disease alone; and the $d$ patient has neither. In the first control group $(\mathrm{G}+5)$, the symptom-disease contingency was set at .5. Specifically, three $a$, one $b$, one $c$, and three $d$ trials were randomly presented in each block. All the subjects had to make a covariation judgment every eight trials, taking into account all the patients seen from the beginning of the session. The second group $(\mathrm{G} 0+5)$ also received a .5 contingency with exactly the same sequence of trials as that for the previous group, but they also received a previous phase in which the contingency between the symptom and the disease was zero. Specifically, in each block, there were two trials of each type $(a, b, c$ and $d)$. The third $(\mathrm{G}-5)$ and fourth $(\mathrm{G} 0-5)$ groups received training similar to that for the previous groups, with the exception that the contingency during the contingent phase was -.5 in each block; specifically, there was one $a$ trial, three $b$ trials, three $c$ trials, and one $d$ trial (see Table 1).

Procedure. Each subject was seated at a distance of $60 \mathrm{~cm}$ from the center of the monitor, on which the instructions and stimuli were presented. The instructions indicated that the subjects had to act as members of a research team from a hospital interested in studying the relationship between the symptom frontopigmentosis and the disease tropical herpiasis. Subsequently, the subjects were informed that a series of photographs of patients would be presented one at a time. Information about symptomatology (the presence or the absence of the symptom) appeared $500 \mathrm{msec}$ before the information about the disease (present or absent) for the same patient. Face duration on the screen was $1,500 \mathrm{msec}$ in each case. After each trial, the subjects had to press a key to see the next face.

Before the experimental trials, each subject had four practice trials, one with each type of patient, in the presence of the experimenter, to resolve any doubt about the task. Then, a scale was shown (ranging from -100 to +100 , with intervals of 5 units). Above the scale, there was a message indicating that the subject had to estimate the strength of the relationship between the symptom and the disease on the basis of all the patients that had been seen up to the moment of the present response.
After the experimenter had made sure that the subjects understood the task, he told the subjects that the experimental trial would begin after he had left the experimental room. The sequence of trials was randomly selected for each subject, with the restriction that, within each block of eight trials, the contingency was the same as that in each whole phase. The task of the subjects was to observe information about patients and make a judgment after every eight trials. The subjects had to move the cursor of the response scale to the point that indicated their estimation of the strength of the relationship between symptom and disease (keeping in mind all the patients seen up to that moment). At the end of the session, the subjects were thanked for their participation.

\section{Results and Discussion}

Figure 1 presents the judgment means in each group after each block of eight trials. It appeared that noncontingency was detected in a similar and accurate way in both the $0+5$ and the $0-5$ groups, which is not surprising, given that they were treated identically. However, this previous experience produced both a clear delay in the detection of subsequent positive contingency, and a facilitation in the detection of the negative one (see Figure 1; also, Table 2), when compared with their respective control $(+5$ or -5$)$ groups.

A $2 \times 4$ (groups $\times$ blocks) analysis of variance (ANOVA) on the judgments of the G0+5 and G0-5 groups during the first phase yielded only an effect of blocks $\left[F(3,90)=4.21, M S_{\mathrm{e}}=3,294.27\right.$; significance level was fixed at .05 for all statistical analyses], but the effect of group and the groups $\times$ block interaction were not significant (both $F \mathrm{~s}<1$ ). The effect of the block factor was due to the fact that the judgment in the first block was higher than that in the rest of the blocks. This preasymptotic positive or excitatory effect is frequently found in both human contingency judgment tasks and animal conditioning studies (Shanks, 1987). However, by the end of this phase, both groups accurately estimated the absence of relationship between the symptom and the disease.

A second $4 \times 4$ (group $\times$ block of trials) ANOVA of the covariation judgments during the second phase showed an effect of group $\left[F(3,60)=21.84, M S_{\mathrm{e}}=67,956.84\right]$ and of its interaction with block $\left[F(9,180)=2.59, M S_{\mathrm{e}}=\right.$ $1,606.81]$, whereas block was only marginally significant 


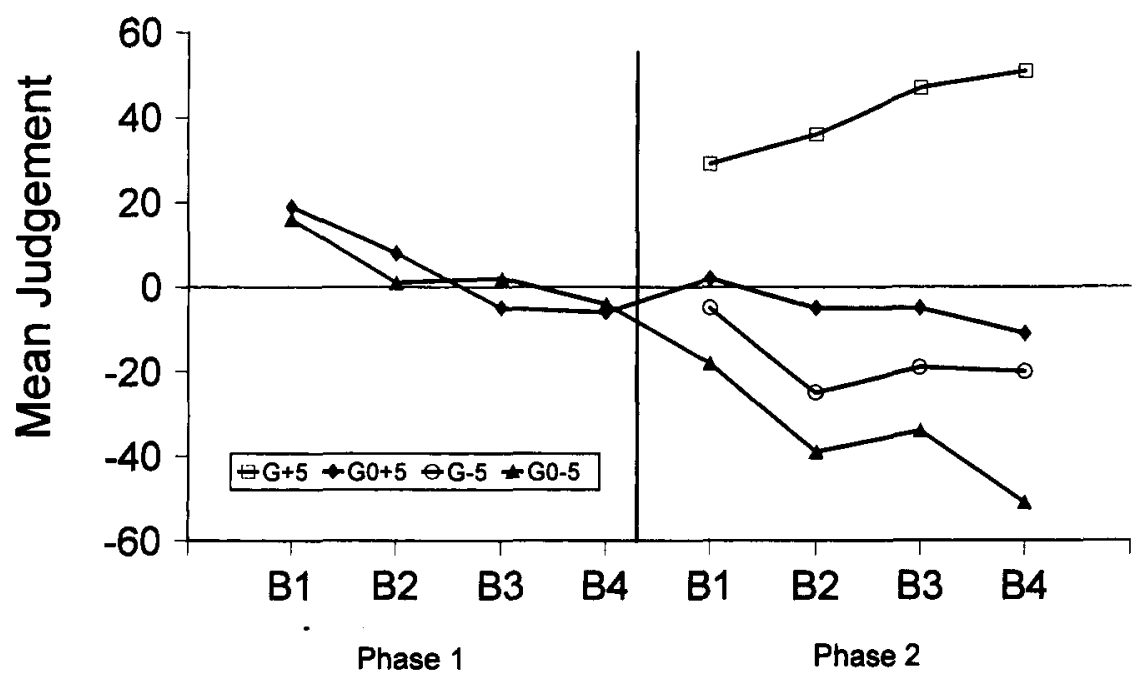

\section{Blocks of Trials}

Figure 1. Mean covariation judgment in each group after each block of eight trials (patients) during both the preexposure phase (Phase 1) and the contingent phase (Phase 2) of Experiment 1.

$\left[F(3,180)=2.39, M S_{\mathrm{e}}=1,482.94, p<.07\right]$. Simple interaction effects analyses using post hoc least significant difference (LSD) tests showed that the $G+5$ and $G-5$ groups' judgments were different from those in the first block, which demonstrated once again that humans are sensitive to different degrees of contingency. The results also showed, first, that the subjects in the $\mathrm{G} 0+5$ group made lower estimations than did the $\mathrm{G}+5$ control group in all four blocks, which indicates that a previous experience with noncontingency between the symptom and the disease produced an impairment in the subsequent detection of positive contingency, in a way similar to the effect of learned irrelevance on subsequent excitatory conditioning. Second, the subjects in the G0-5 groups made judgments that were lower than the estimations from the $G-5$ group in the second block. This unexpected effect indicates a facilitation in the subsequent detection of negative contingency as a result of the previous noncontingent experience, but this facilitation was due to the poor detection of the negative contingency in the control group.

These results showed the asymmetrical effect of a noncontingent experience on the detection of subsequent positive and negative contingency, inasmuch as a similar noncontingent preexposure produced both a delay in the detection of a subsequent positive contingency and a facilitation of a negative one. This last result appears to be different from the effect of learned irrelevance on inhibitory conditioning. However, before we considered explanations for this asymmetry and for the differences with learned irrelevance, the next logical step was to replicate this asymmetrical effect with different levels of positive and negative contingency during the second phase, to further test its reliability.

\section{EXPERIMENTS 2A AND 2B}

In the first experiment, the demonstration of an asymmetrical effect of a noncontingent experience on the subsequent detection of positive or negative covariations was made, using only an intermediate level of contingency (.5). Therefore, Experiments $2 \mathrm{~A}$ and $2 \mathrm{~B}$ attempted to replicate this effect with different levels of positive and negative contingency (ranging from -.75 to .75 ).

\section{Method}

Subjects and Apparatus. Two groups of 96 undergraduates from introductory psychology classes at the University of Granada participated in these experiments. The computer, programs, and stimuli were the same as those used in Experiment 1.

Design and Procedure. The instructions given, the details of presentation of the trials, and the elicitation of judgments were the same as those in Experiment 1. Each subject was randomly assigned to one of the groups described in Table 2 for Experiments $2 \mathrm{~A}$ and $2 \mathrm{~B}$.

The contingent groups $(+25,+50$, and +75 in Experiment $2 \mathrm{~A}$ and $-25,-50$, and -75 in Experiment $2 B$ ) received only a contingent phase, according to their contingency levels. However, in the noncontingent groups $(0+25,0+50,0+75$ in Experiment $2 \mathrm{~A}$ and $0-25$, $0-50$, and $0-75$ in Experiment $2 B$ ), all the participants received a prior noncontingent phase, identical to the one experienced by the noncontingent groups in Experiment 1, before estimating their assigned contingency between the symptom and the disease during the second phase. All the other procedural details were identical to those described in Experiment 1.

\section{Results and Discussion}

Table 2 shows the judgment means for each group in Experiments $2 \mathrm{~A}$ and $2 \mathrm{~B}$ during the second phase.

A $2 \times 3 \times 4$ ANOVA (preexposure $\times$ contingency $\times$ block) was performed in each experiment on the judgments of the subjects during the second correlated phase. 
Table 2

The Mean Covariation Judgment in Each Group of Experiments 1, 2, and 3 After Each of the Four Blocks of Eight Trials (Patients) in Each Phase

\begin{tabular}{|c|c|c|c|c|c|c|c|c|c|c|}
\hline & \multicolumn{5}{|c|}{ Phase 1} & \multicolumn{5}{|c|}{ Phase 2} \\
\hline & B1 & B2 & B3 & B4 & $M$ & B1 & B2 & B3 & $\mathrm{B} 4$ & $M$ \\
\hline \multicolumn{11}{|c|}{ Experiment 1} \\
\hline $\mathrm{G}+5$ & & & & & & 29 & 36 & 47 & 51 & 41 \\
\hline $\mathrm{G} 0+5$ & 19 & 8 & -5 & -6 & 4 & 2 & -5 & -5 & -11 & -5 \\
\hline$G-5$ & & & & & & -5 & -25 & -19 & -20 & -15 \\
\hline $\mathrm{G} 0-5$ & 16 & 1 & 2 & -4 & 4 & -18 & -39 & -34 & -51 & -36 \\
\hline \multicolumn{11}{|c|}{ Experiment $2 \mathrm{~A}$} \\
\hline $\mathrm{G}+25$ & & & & & & 26 & 28 & 40 & 33 & 32 \\
\hline $\mathrm{G} 0+25$ & 6 & 5 & 5 & 12 & 7 & 14 & 16 & 16 & 22 & 17 \\
\hline $\mathrm{G}+50$ & & & & & & 40 & 42 & 52 & 56 & 48 \\
\hline $\mathrm{G} 0+50$ & -1 & -7 & -8 & -12 & -7 & 7 & 8 & 10 & 15 & 10 \\
\hline $\mathrm{G}+75$ & & & & & & 66 & 52 & 62 & 56 & 59 \\
\hline $\mathrm{G} 0+75$ & 24 & 4 & 13 & 20 & 15 & 39 & 47 & 50 & 49 & 46 \\
\hline \multicolumn{11}{|c|}{ Experiment $2 \mathrm{~B}$} \\
\hline$G-25$ & & & & & & -6 & -28 & -10 & -28 & -18 \\
\hline G0 $0-25$ & -15 & -10 & -20 & -1 & -11 & -16 & -16 & -27 & -39 & -25 \\
\hline $\mathrm{G}-50$ & & & & & & -6 & -9 & -26 & -24 & -16 \\
\hline $\mathrm{G} 0-50$ & -3 & -7 & -7 & -4 & -5 & -34 & -43 & -51 & -50 & -45 \\
\hline$G-75$ & & & & & & -19 & -48 & -43 & -47 & -39 \\
\hline G0-75 & 5 & -10 & 1 & 4 & 0 & -39 & -55 & -61 & -58 & -53 \\
\hline \multicolumn{11}{|c|}{ Experiment 3} \\
\hline $\mathrm{G}+5 \mathrm{C}$ & & & & & & 27 & 32 & 30 & 23 & 28 \\
\hline $\mathrm{G} 0+5 \mathrm{C}$ & -8 & -7 & -20 & -18 & -13 & 2 & 3 & 15 & 13 & 8 \\
\hline$G-5 C$ & & & & & & -9 & -12 & -23 & -26 & -18 \\
\hline $\mathrm{G} 0-5 \mathrm{C}$ & 0 & 1 & -2 & 1 & 0 & -9 & -14 & -13 & -13 & -12 \\
\hline
\end{tabular}

Note-In each experiment, $\mathrm{G}$ stands for group. The number in each group indicates the contingency $(+5=.5 ;-5=-.5 ; 0=$ no contingency), as explained in Table 1 . B indicates block number, with each block containing eight trials. In Experiment 3 , the $C$ in each group means the existence of the added cue in each trial, to simulate the role of the context. Data are rounded to the nearest integer value. $M$ is the mean of the four blocks.

In Experiment 2A, the ANOVA revealed a main effect of all three factors [preexposure, $F(1,90)=18.26, M S_{\mathrm{e}}=$ $60,600.14$; contingency, $F(2,90)=8.53, M S_{\mathrm{e}}=28,315.02$; and block, $\left.F(3,270)=5.78, M S_{\mathrm{e}}=1,349.27\right]$, but no interaction was found to be significant.

The effect of preexposure indicated a similar impairment on the detection of the positive contingency during the second phase in each noncontingent group, with respect to its control contingent group, which replicated the results of the previous experiment. The statistical analysis of the main effect of contingency with a LSD test showed differences between the +.75 condition and the other two contingency conditions $(+25$ and +5$)$, which did not differ significantly. The analysis of block is not presented, as it does not add any relevant information, inasmuch as acquisition functions were not clearly evident in these experiments, unlike in Experiment 1.

A $2 \times 3 \times 4$ ANOVA (preexposure $\times$ contingency $\times$ block) for Experiment $2 \mathrm{~B}$ also revealed that the three main factors were significant [preexposure, $F(1,90)=$ 5.02, $M S_{\mathrm{e}}=25,350.00$; contingency, $F(2,90)=4.11$, $M S_{\mathrm{e}}=20,766.47$; and block, $F(3,270)=14.68, M S_{\mathrm{e}}=$ 7,665.61], whereas no interaction was significant. Experiment $2 \mathrm{~B}$ replicated the decremental effect of preexposure making the estimations more negative than was previously observed in Experiment 1, thereby again indicating a similar facilitation of the detection of negative contingency in all of the noncontingent groups. The statistical analysis of the main effect of contingency with the LSD test only revealed significant differences between the -.25 contingency condition and the other two conditions $(-.50$ and -.75$)$.

These results replicated the asymmetrical effect found in Experiment 1, as they demonstrated that a previous noncontingent experience produced both an impairment in the detection of a subsequent positive contingency (Experiment 2A) and an improvement when it was negative (Experiment 2B) in all groups; this result was independent of the level of contingency during the second phase, as no interaction was found to be significant.

As was stated before, statistical and normative models can explain the decremental effect on the detection of positive contingency by assuming that subjects calculate the contingency between events on the basis of all the patients seen from the beginning of training, including the patients seen during the first phase, as they were instructed to do before the task. However, with this same assumption, they cannot explain the more negative judgments on the detection of subsequent negative contingency, which raises doubts about the rationality of the 
human estimations. Moreover, neither theories based on attention and associability decrements nor those based on memory demands (Shaklee \& Mims, 1982) can explain the asymmetrical effect of previous noncontingency, because covariation judgments should be systematically worse and less accurate under these attention or memory conditions. In addition, a different study, using a procedure that was the same as that used in these experiments, replicated the asymmetrical effect of previous noncontingency on subsequent contingency detection and found this effect only when the events were the same in both phases. This refutes any interpretation of the effects as merely being due to the prior experience with the judgment scale in the noncontingent groups (Maldonado, Catena, García, \& Cándido,1996).

Two different approaches, however, could explain this asymmetrical effect. An associative model, such as the one proposed by Rescorla and Wagner (1972) and currently used to explain human covariation learning (Allan, 1993; Shanks, 1995), can accommodate these effects by assuming that the context - or another possible cue - acquires excitatory strength during the uncorrelated presentations of the symptom and the disease. This context-associative strength should block the subsequent acquisition of excitatory strength of the irrelevant cue, whereas it should enhance the acquisition of inhibition, when compared with that of a naive group, if the training procedure were just a positive or negative correlation between the cue and the consequence in the same context in which preexposure occurred. In the naive condition, the associative strength of the cue and the context should be zero at the beginning of the correlated phase. Therefore, if associative strength maps onto human contingency judgment, this model can account for the observed asymmetrical effects.

Alternatively, a recent belief revision model (Catena, Maldonado, \& Cándido, 1998), proposed as an explanation of the frequency-of-judgment and sequential effects, could also explain the asymmetrical effect of previous noncontingency. This model is based on the well-documented effect that, in human covariation detection tasks, the weight of each type of trial in defining the contingency is different (see Crocker, 1982; Kao \& Wasserman, 1993; Levin, Wasserman, \& Kao, 1993; Wasserman, Dorner, $\&$ Kao, 1990). The influence of the weights can be ordered as follows: $a>c \geq b>d$, with $a$ and $d$ being cells that favor positive contingency, and $c$ and $b$ cells that favor negative contingency. This model assumes that people use a simple rule, such as a weighted $\Delta D$ rule, to compute the contingency (see the Appendix), with the trial type subjective value being positive and higher for $a$ trials than for $d$ trials and negative and similar for $b$ and $c$ trials. However, if the subjects compute covariation on the basis of all the evidence from the beginning of training with this simple rule, the results will be similar to those obtained from a nonweighted rule or normative models (see Table 1). But the model proposed also includes a second mechanism that acts each time a judgment is asked of the subject and that serves to update the new evidence obtained from the last judgment. This second mechanism allows acquisition effects and sequential learning. In this way, this model can also explain the effect of a noncontingent preexposure, provided that we also assume that the weights usually granted to each type of trial can change because of this experience.

Accordingly, we assumed that, after the noncontingent preexposure, the weight of the type of trials should change when computing the new evidence (see the Appendix). Hence, during contingent training, when subjects are again asked about the relationships between two events after noncontingent preexposure, judgment updating will be sensitive to the subjective previous experience, which will especially reduce the weight of the confirming positive trials (i.e., the $a$ trials; see the General Discussion section and the Appendix for a detailed discussion and a simulation of the predictions of the model). In this way, this model, as well as associative models, can explain the observed asymmetrical effect. The next two experiments tested this model and contrasted it with associative models.

\section{EXPERIMENT 3}

Associative models assume that, when people experience the absence of correlation between a cue and a consequence, the context acquires excitatory associative strength and should then block subsequent excitatory conditioning of the cue. But these models also predict more negative judgments on the detection of subsequent negative contingency, because the excitatory strength acquired by the context should induce more inhibitory strength in the negatively correlated cue if the context remains the same. If positive and negative associative strength maps onto covariation judgments, a previous noncontingency should produce lower judgments (an impairment) in the detection of subsequent positive contingency but more negative judgments (a facilitation) on the detection of subsequent negative contingency, when compared with subjects without this uncorrelated experience, as was found in Experiments 1 and 2. However, the concept of context in a human diagnostic task is vague and not easily represented, but we can make explicit the existence of another possible cue by the addition of a new symptom that plays the role of the context assumed in associative models. Therefore, in Experiment 3, the design and the procedure were similar to those of Experiment 1 , except that a new symptom, playing the possible role of the context, was presented in every trial (each patient), in order to test the predictions derived from the associative model proposed as an explanation for the asymmetrical effects of previous noncontingency.

\section{Method}

Subjects, Apparatus, and Procedure. The subjects were 64 undergraduates from introductory psychology classes of the University of Granada. The computers and programs were the same as those used in the previous experiments.

We used the four conditions described in Experiment $1(\mathrm{G} 0+5 \mathrm{C}$, $\mathrm{G}+5 \mathrm{C}, \mathrm{G} 0-5 \mathrm{C}$, and $\mathrm{G}-5 \mathrm{C}$ ), with only two differences. The first was that the symptoms appeared as printed words in a right panel 
of the screen, while in the left panel there still appeared a face to inform the subject that there was a new patient. In this way, it was possible to inform the subject about the target symptom (pigmentosis, symptom A) and the new symptom (brucesis, the added symptom $C$, which was present on all the trials and played the role of context), before informing the subject about the disease (herpiasis).

Therefore, the four types of trials in this experiment were as follows: type $a(\mathrm{CA}+)$, in which the two symptoms and the disease appeared; type $b\left(\mathrm{CA}^{-}\right)$, in which the patient had only the two symptoms; type $c(\mathrm{C}+)$, in which the patient suffering the disease had only the added symptom; and type $d(\mathrm{C}-)$, in which only the added symptom was presented.

The instructions were the same as those in Experiment 1. However, the subjects were informed before treatment that their task was only to estimate the relationship between symptom A (i.e., pigmentosis) and the disease (i.e., herpiasis) as in Experiment 1, although they were also informed that another symptom could appear on every trial.

The manipulation of contingency with respect to symptom A was done exactly as in Experiment 1 for each group (see Table 1), but with the inclusion of symptom $C$ in all trial types, which generated for symptom $A$ the same contingencies experienced by each group in Experiment $1(\mathrm{G} 0+5, \mathrm{G}+5, \mathrm{G} 0-5$, and $\mathrm{G}-5)$.

The second difference was that this experiment was done with only two groups. The first group experienced the task twice in counterbalanced order, once with only the positive contingency $(+5)$ and the other time with the uncorrelated phase first $(0+5)$. Similarly, the second group experienced the task once with only the negative contingency $(-.5)$ and once with both the uncorrelated and the negatively correlated phases $(0-5)$. In one task, we used the previously described symptoms (pigmentosis and brucesis as the added symptom) and disease (herpiasis). In the other task, the symptoms were mebiasis and pirrosis, respectively, and the disease was called neuplesis. All the symptoms were presented counterbalanced with respect to task and condition. The rest of the procedural details were the same as those in the first experiment.

\section{Results and Discussion}

The judgments of both groups during preexposure suggest that the subjects detected no contingency in the absence of a contingency between symptom $A$ and the disease in the presence of the added cue (see Table 2). A $2 \times 4$ (group $\times$ block) ANOVA of the judgments during the uncorrelated phase in both groups did not yield any significant difference, as was expected [group, $F(1,62)=$ $2.20, M S_{\mathrm{e}}=5,079.33$; block, $F(3,186)=1.70, M S_{\mathrm{e}}=$ 624.62 ; and their interaction, $F(3,186)=1.01, M S_{\mathrm{e}}=$ 624.62].

The results obtained during the correlated phase in each condition (see, also, Table 2) appear to show, first, that the subjects with previous noncontingent experience made lower estimations of the subsequent positive contingency than did naive subjects. However, the same preexposure did not reveal any reliable effect on the detection of negative contingency, relative to the absence of such preexposure.

In the analysis of the contingency estimations between symptom $A$ and the disease during the contingent phase, a $2 \times 2 \times 2 \times 4$ (contingency or group, order, preexposure, and block) ANOVA was used. The effect of order was not significant, either alone $\left[F(1,60)=1.42, M S_{\mathrm{e}}=\right.$
$8,723.27, p<.24]$ or in any interaction with the other factors (all $F \mathrm{~s}<1$ ), except for the contingency $\times$ order interaction $\left[F(1,60)=3.69, M S_{\mathrm{e}}=8,723, p<.07\right]$, which showed that this factor had no influence on the results obtained. However, the same analysis revealed a main effect of contingency $\left[F(1,60)=15.02, M S_{\mathrm{e}}=8,723.27\right]$ and also an interaction between contingency and preexposure $\left[F(1,60)=7.73, M S_{\mathrm{e}}=2,634.70\right]$. Finally, there were interactions between contingency and blocks $\left[F(3,180)=4.39, M S_{\mathrm{e}}=394.66\right]$ and preexposure and block $\left[F(3,180)=3.43, M S_{\mathrm{e}}=671.96\right]$; all other effects and interactions were not significant. We analyzed only the significant effects of contingency and its interaction with preexposure, because the interactions between block and the other factors were not relevant to the focus of this experiment.

The effect of contingency showed that the subjects were able to accurately detect the differences in contingency $(+.5$ and -.5$)$, despite the addition of another cue (C). However, the most important and interesting result was that the interaction between contingency and preexposure was significant. As in the first experiment, post hoc LSD tests demonstrated that there were significant differences between positive and negative contingency conditions, as both the $\mathrm{G} 0+5 \mathrm{C}$ and the $\mathrm{G}+5 \mathrm{C}$ groups differed from either the $\mathrm{G} 0-5 \mathrm{C}$ or the $\mathrm{G}-5 \mathrm{C}$ group. LSD tests also yielded differences in the detection of positive contingency between the $\mathrm{G} 0+5 \mathrm{C}$ and the $\mathrm{G}+5 \mathrm{C}$ groups, thereby indicating an impairment from the prior noncontingent preexposure of the $\mathrm{G} 0+5 \mathrm{C}$ group. This result was similar to that found in Experiment 1, as the judgments with the $\mathrm{G} 0+5$ conditions were lower than those of the $\mathrm{G}+5$ groups.

Finally, this same preexposure did not produce any significant difference between both negative conditions (G0-5C and $\mathrm{G}-5 \mathrm{C}$ ) on the detection of negative contingency. This last effect was different from that obtained in Experiments 1 and 2, which suggests that the more negative judgments previously found in the detection of negative contingency between single cues after noncontingent preexposure tend to disappear when multiple cues are used. These results cast doubts on the role of the context, assumed by associative models to explain the previous findings.

To summarize, the results of this experiment suggest that the facilitated detection of negative contingency after a previous noncontingent experience with the same events cannot be attributed to an effect of an excitatory context. This is contrary to accounts of associative models of the asymmetrical effect of noncontingency that is found with single cues. Nor can these models explain the delay of a subsequent inhibitory conditioning produced by learned irrelevance (Baker \& MacKintosh, 1977). We will return later to a discussion of this issue.

As was stated before, humans appear to assign different weights to each type of trial when they estimate the actual contingency between two events (Kao \& Wasser- 
man, 1993). This differential weighting effect probably reflects the influence of prior beliefs and the possible existence of specific biases in their processing, owing to previous experience, among other factors (Miller \& Matute, 1996). For example, Matute, Arcediano, and Miller (1996) found strong biases concerning which foods were most apt to act as causes of an allergic reaction, which suggests the existence of specific biases acquired through previous direct or indirect experience with these foods. This processing bias can be captured by differential weighting, especially of the type $a$ trials, when computing the contingency between each element with the food.

Therefore, if we assume that a previous noncontingent experience alters the processing of these stimuli (Bennet et al., 1995), this preexposure should change the weights assigned to each type of trial. The next two experiments tested these predictions, derived from the Catena et al. (1998) model proposed as an explanation for the asymmetrical effect (see the Appendix).

\section{EXPERIMENTS 4A AND 4B}

The differential value of each trial type on contingency detection tasks is a known effect (see Crocker, 1982; Levin et al., 1993; Wasserman et al., 1990), and its influence can be ordered as follows: $a>c \geq b>d$. In fact, recent models based on so-called nonnormative strategies give different but fixed weights to each type of trial either with cognitive rule-based mechanisms (Kao \& Wasserman, 1993) or with associative mechanisms (Tassoni, 1995; Van Hamme \& Wasserman, 1994).

The main assumption of the next two experiments was that a previous noncontingency experience would alter these weights, especially producing a lesser influence of the positive cases (the $a$ type trials). This change in the weight of different types of events could explain both the facilitation and the delay in the detection of subsequent positive and negative contingency, respectively, if an averaging model based on cognitive strategies were used to explain actual judgments (Catena et al., 1998; see the Appendix).

Consequently, the aim of these experiments was to determine whether the subjects assign different weights to each kind of trial used to define the contingency between two events in our diagnostic task and, if so, whether these changes reduce the weight of the positive cases (the $a$ trials).

\section{Method}

Subjects, Apparatus, and Procedure. The subjects were 205 (112 in Experiment 4A and 93 in Experiment 4B) undergraduates from introductory psychology classes at the University of Granada. The computers and programs were the same as those used in the previous experiments. The stimuli used for Experiment 4A were the same as those used in Experiment 1, whereas for Experiment 4B, we used printed words presented as in Experiment 3.

To determine the empirical weights of the different types of trials, all the subjects were asked to give, with paper and pencil, the values they assigned to the four different types of patients presented (it is important to note that verbally assessed weights might differ from effective weights). For this purpose, they had to note, on a scale ranging from -7 to +7 ( -7 anchored by very much negative change, +7 by very much positive change, and 0 by nothing, which were written immediately under the scale), how much their judgments about the degree of relationship between the symptom and the disease would change and in which direction, after having seen each type of patient $(a, b, c$, and $d$; order counterbalanced within each group). For example, the translation into English of the question for the type $a$ patients was: "Imagine that you see a patient suffering from both the symptom and the disease. Note on the scale how much your judgment would change and in which direction."

In Experiment 4A, two groups were used. The subjects in the noncontingent group (G0) had to answer the questions after they had completed the noncontingent task (i.e., after having seen 32 patients, as in the similar groups in Experiments 1 and 2). The subjects in the naive group completed the same sheet after the four practice trials, one of each type (i.e., $a, b, c$, and $d$ ), and after having used the contingency scale, but before they received the contingent phase of training. Half the subjects in each group were subsequently exposed to 32 new patients with either a positive or a negative contingency, as in Experiment 1.

In Experiment 4B, there were three groups, which experienced noncontingency $(\mathrm{G} 0)$, a positive .5 contingency $(\mathrm{G}+5)$, or a negative .5 contingency $(\mathrm{G}-5)$ between the symptom (pigmentosis) and the disease (herpiasis) in 32 patients, presented in printed form, as in Experiment 3. In each group, the subjects filled out the same sheet twice in a counterbalanced manner: first, after the four practice trials, as explained before, and after having used the contingency scale, but before the experimental trials began, and, then, after they had completed the task (i.e., after having seen 32 patients).

\section{Results and Discussion}

Table 3 shows the weights assigned to each type of trial either before or after a noncontingency experience in Experiments 4A and 4B. They indicated first that, according to the proportion of change, the type of trial can

Table 3

Evaluation of Each Type of Trial in Each Group of Experiments $4 A$ and $4 B$

\begin{tabular}{|c|c|c|c|c|}
\hline \multirow[b]{2}{*}{ Group } & \multicolumn{4}{|c|}{ Type of Trial Evaluation } \\
\hline & $a$ & $b$ & $c$ & $d$ \\
\hline & \multicolumn{4}{|c|}{ Experiment 4A } \\
\hline Naive & 3.3 & -1.8 & -2.2 & 0.7 \\
\hline \multirow[t]{2}{*}{ Preexposure } & 1.9 & -1.4 & -0.9 & 0.3 \\
\hline & \multicolumn{4}{|c|}{ Experiment 4B } \\
\hline \multicolumn{5}{|l|}{$\mathrm{G}+5$} \\
\hline Preexposure & 3.7 & -1.8 & -2.6 & 0.7 \\
\hline Postexposure & 3.6 & -1.7 & -2.7 & 0.7 \\
\hline \multicolumn{5}{|l|}{ G0 } \\
\hline Preexposure & 4.1 & -1.8 & -2.6 & 0.3 \\
\hline Postexposure & 2.3 & -2.1 & -2.8 & 0.5 \\
\hline$G-5$ & & & & \\
\hline Preexposure & 3.8 & -0.5 & -2.4 & 0.0 \\
\hline Postexposure & 2.4 & -1.6 & -2.1 & 0.0 \\
\hline
\end{tabular}

Note-Trial type ( $a, b, c$, and $d$ ) indicates whether the symptom and the disease were present or absent in each patient, as is explained in the text. The question was asked in Experiment 4A before any experience in the naive group and after a noncontingent preexposure in the preexposure group, whereas in Experiment 4B, it was asked before any experience (preexposure) and after 32 patients (postexposure) in each group had been seen. The number after the $\mathrm{G}$ indicates the contingency between the symptom and the disease in each group $(+.5,0$, or -.5$)$. 
be ordered as $|a|>|c| \geq|b|>|d|$. Second, they showed that a noncontingent experience tends to reduce the differences between them, especially by reducing the weight assigned to the type $a$ trials.

In Experiment $4 \mathrm{~A}$, the $2 \times 4$ mixed ANOVA (group $X$ trial type) revealed an effect of trial type $[F(3,324)=$ $\left.68.61, M S_{\mathrm{e}}=447.96\right]$ and of its interaction with group $\left[F(9,369)=5.59, M S_{\mathrm{e}}=36.50\right]$.

Post hoc LSD tests indicated that, with and without noncontingent treatment, trial type $a$ was weighted more heavily that of types $b, c$, and $d$; trial type $d$ was also higher than $b$ and $c$, and there were no appreciable differences between the last two types of trial. However, there were differences between the two groups in the weights of the type $a$ trials $\left[F(1,110)=9,69, M S_{\mathrm{e}}=54.32\right]$, and also in the type $c$ trials $\left[F(1,110)=5,51, M S_{\mathrm{e}}=43.75\right]$, but not in $b$ or $d$ trial types (both $F \mathrm{~s}<1$ ), thereby reflecting an effect of preexposure (see Table 3 ). These results indicate that a prior noncontingent experience can produce a reduction in the weights of each type of trial, significantly so for trial $d$ type and particularly for trial type $a$. Experiment 4B used a within-subjects design to assess the generality of these changes in trial type $a$ weights.

In Experiment 4B, because of a significant violation of the variance-covariance equality assumption, Wilcoxon matched-pairs tests were performed to compare the within-subjects estimations of trial-type weights. The results showed that there were no significant differences between the weights of each type of trial after the contingency judgment task was completed, except in the case of the type $a$ trials in the noncontingent group (G0), in which there was a significant reduction $(p<.02)$, although the reduction in the negative contingent group $(\mathrm{G}-5)$ in the type $a$ trials was also marginally significant $(p<.11)$. It is important to keep in mind that the subjects in Experiment 4B had to fill out questionnaire sheets twice and probably remembered what they had done on the first sheet when filling out the second one, which could have reduced the effect.

In this experiment, as in Experiment 4A, comparisons of the evaluations of the influence of each trial type on each group before and after the covariation learning experience revealed, that the influence of the type $a$ trials was estimated as positive and higher than those of trial types $d, b$, and $c$ in the three groups and in both phases (all ps $<.03$ ). The evaluation of trial type $d$ was also positive and higher than that of $b$ and $c$ (in all comparisons, $p<.022$, except in the $\mathrm{G}-5$ group, in which $d$ and $b$ were not significantly different either before, $p<.71$, or after, $p<.07$, the learning experience), whereas the evaluations of trial types $b$ and $c$ were negative and did not differ between themselves (all $p$ s $<.07$ ), except in the preevaluation of the $\mathrm{G}+5(p<.05)$ and $\mathrm{G}-5(p<.004)$ groups, in which the influence of the type $b$ trials was rated as less negative than that of the type $c$ trials.

To summarize, the results of Experiments 4A and 4B replicated previous findings about the weights of each trial type in contingency judgment tasks (Kao \& Wasser- man, 1993; Wasserman et al., 1990). It is important to note that the absolute value given to each type of trial as the proportion of change produced in the contingency judgment was $a>c \geq b>d$, which is also similar to that found with different methods or scales (Kao \& Wasserman, 1993). At the same time, the results revealed that $a$ and $d$ trials induced a positive evaluation, whereas $c$ and $b$ trials induced a negative evaluation, which replicates previous findings.

The most important finding, however, was that, in both Experiment $4 \mathrm{~A}$ and Experiment $4 \mathrm{~B}$, the previous noncontingent experience reliably reduced the weight of the $a$ cases. Experiment 4B also revealed that this noncontingent experience reduced the assigned weight only in the case of the $a$ trials; no reliable change was apparent in the rest of the trial types. In addition, negative contingency training produced a marginally significant reduction in the weights assigned to the type $a$ trials, and it also produced a nonsignificant tendency toward an increased negative weight for type $b$ trials. Finally, in the positive contingency group, there was no appreciable change at all in any of the weights assigned to any type of trial.

These results collectively demonstrate that a noncontingent experience produces a reduction of the weights of type $a$ trials. This finding supports the larger conclusion that trial type weights are not fixed; they can change as a function of experience. This change can account for the delay as well as the facilitation effects found in these experiments, an issue to which we will return in the General Discussion section. Our results illustrate, once again, the importance of prior beliefs and trial order (sequence) effects, and our belief revision model demonstrates one way in which a statistical (i.e. nonassociative) model might account for such sequence effects.

\section{GENERAL DISCUSSION}

The results of these experiments have shown that prior experience with two uncorrelated events produced two opposite effects in the subsequent detection of positive and negative contingency between the same eventsspecifically, a delay when the contingency was positive and facilitated negative judgments (Experiments 1 and 2).

This asymmetrical effect is not predicted by any of the statistical models - those that use unconditional probabilities (see Table 1), those that use conditional probabilities (e.g., the focal set model of Cheng \& Novick, 1992), or the more recent power pc model (Cheng, 1997) — that are currently used to explain human covariation detection. However, these models can explain the lower positive judgments after the noncontingent preexposure, by the assumption that the subjects applied their statistical rule from the beginning of training when estimating the relationship between the symptom and the disease. But this approach predicts that the judgments of the preexposed subjects should also be less negative in the detection of negative contingencies. That is, if they were taking 
into account all the patients seen up to the moment of judgment, just the opposite results to those found would be expected, which makes it difficult to explain the asymmetrical effect as being a result of applying any single statistical rule when computing the current contingency.

It is important to note that this asymmetrical effect was found by using a procedure in which noncontingency was created with $p$ (disease/symptom) $=.5$ and $p$ (disease/no symptom $)=.5$. However, it has also been shown that the detection of noncontingency is affected by factors such as trial order, the absolute probability of signaled reinforced trials, and the percentages of consequences (i.e., outcome density) received during training (Baker, Berbrier, \& Vallée-Tourangeau, 1989; Benedict, 1991; Matute, 1995; Shanks, 1985, 1987). Whether such factors also differentially affect the subsequent detection of positive and negative contingencies (i.e., create an asymmetry) can be determined only through further research.

The facilitation of the detection of negative contingency differed from the effect of learned irrelevance on subsequent inhibitory conditioning (Baker \& MacKintosh, 1977), but our data may not be relevant to conditioned inhibition, as the usual procedure of Pavlov for conditioned inhibition $(\mathrm{A}+, \mathrm{AB}-)$, which was also used by Baker and MacKintosh (1977), implies a positive contingency (i.e., an excitatory conditioning) between cue $\mathrm{A}$ and the outcome $(+)$, in order to induce inhibitory properties into the negatively correlated stimulus $B$ when both cues are presented without the outcome $(\mathrm{AB}-)$. Therefore, it is possible that the differences in the specific procedure could also explain the differences between learned irrelevance and our results.

In fact, the different results obtained in the detection of negative contingencies and conditioned inhibition, using different training procedures, accords with the proposal of several authors - for example, Hallam, Matzel, Sloat, and Miller (1990), who concluded that the expression of conditioned inhibition and the magnitude of the conditioned inhibitory response to a stimulus depends on the procedure used to acquire inhibition. Moreover, they also argued that inhibitory conditioning is a complex phenomenon that usually involves multiple associations and that both a summation test and delay are necessary to distinguish conditioned inhibition from changes in attention to the putative inhibitor. All these differences suggest that different mechanisms or cognitive rules were probably acting when the contingencies with single (explicitly unpaired procedure) and multiple (Pavlovian procedure) cues were computed, and they also suggest that conditioned inhibition cannot be reduced to the mere computation of the negative correlation between a signal and a consequence (Allan, 1993; see Williams, 1996, for a detailed discussion of the differences between conditioned inhibition and negative covariation judgments). A second possible source of the discrepancy could arise from differences in how conditioned inhibition and nega- tive contingency are assessed - that is, by behavioral summation and delay tests, as opposed to verbal judgment.

The problem remains to explain the asymmetrical effect of noncontingency when estimating the subsequent contingency between single cues. Associative models could accommodate this effect by the assumption that the context or any other possible cause acquires positive strength during the uncorrelated phase and then has its influence during subsequent contingent training as a result of cue competition (Holland \& Rescorla, 1982; Maldonado et al., 1991). With this assumption, the context should impair the subsequent acquisition of excitation by the irrelevant cue, whereas it should facilitate its acquisition of inhibition.

Experiment 3 tested this associative prediction by the addition of a new symptom in all types of trials. The results demonstrated, once again, an impairment in the subsequent detection of positive contingency. However, the addition of the new cue abolished the differences between naive and preexposed groups in the subsequent detection of negative contingencies, which did not confirm the enhanced facilitatory effects predicted by associative models.

Toward explaining the present asymmetry, Experiments 4A and 4B tested a recent statistical model (Catena et al., 1998), which they call the belief revision model. The results of these experiments showed that the weights assigned by the subjects to each type of trial differed, with the $a$ cases being positive and more influential than the $b$ and $c$ ones, the influence of which was rated as negative, whereas the influence of the $d$ cases was also estimated as positive but with less influence on judgments than type $a$ trials. Moreover, these experiments also showed that the trial weights can change as an effect of previous uncorrelated experience, mainly seen in a reduction of the weight of the positive $a$ cases. In order to capture the differential influence of each type of trial, Markman (1989) proposed a modification of the Rescorla and Wagner (1972) rule, in which it is possible to code the rate parameters for cue present and absent and for outcome present and absent, so that they capture the weights that subjects assign to each class of trial. Moreover, within this associative connectionist framework, Markman suggested giving to the learning parameter a negative value when the cue is absent, to capture the effect of the negative contingency judgments (i.e., his choice, like that of the belief revision model, was empirically driven). As Markman stated (see, also, Dickinson \& Burke, 1996; Tassoni, 1995; Van Hamme \& Wasserman, 1994), an absent cue provides information about correlation mainly when its presence is expected in the experimental context. If the absence of the outcome is given a negative value, each cell can have a different and potentially nonzero impact on the cueoutcome covariation judgment, as our subjects reported (Experiment 4). Although these models appear to be more powerful than that of Rescorla and Wagner, they are still unable to explain recent findings on the effect of the fre- 
quency of judgment (Catena et al., 1998), and they are also unable to explain the asymmetrical effect of prior noncontingency because they assume that there are fixed learning parameters (i.e., fixed weights), whereas our data indicate that the weights can change between trials.

According to the Catena et al. (1998) model, however, there is an information integration system that is activated each time the subject is asked to judge the relationship between events. This system behaves according to Equation 1:

$$
J_{i}=J_{i-1}+\beta\left(\text { NewEvidence }-J_{i-1}\right),
$$

in which $J_{i}$ stands for the current judgment, $J_{i-1}$ is the prior judgment, and $\beta$ is a growth rate parameter, the value of which will only affect the trialwise adjustment to the objective contingency, thereby accounting for acquisition and certain sequential effects. Finally, NewEvidence is the subjective value of the evidence presented between two consecutive responses or judgments (see the Appendix).

This equation suggests that people must keep in memory only the last judgment about the relationships between the cue and the consequence, the current weight of each trial type, and the new evidence since the last judgment. This is a distinct departure from other statistical models (e.g., Rescorla, 1968), in which all the prior trials are used to compute covariation. The term $J_{i-1}$ summarizes all previous experience and prior beliefs about the task, and the new evidence is contrasted with it. Obviously, NewEvidence is the key concept in the equation, and it refers to the new experience with the task or with the events that have occurred since the last response or the last judgment during the last block of trials. Our assumption is that, at least in the detection of contingency between pairs of events or when the data are presented in a summary table, the value assigned to this NewEvidence relies on the computation of a statistical rule.

The model proposes that people compute NewEvidence by using the information from all types of trials since the last evaluation on the basis of trial type frequencies multiplied by weights, with weights being positive for $a$ and $d$ trials and negative for $b$ and $c$ trials, as in a weighted $\Delta D$ rule, expressed in Equation 2 (Catena et al., 1998; see, also, the Appendix):

$$
\text { NewEvidence }=\left(w_{a} A-w_{b} B-w_{c} C+w_{d} D\right) / N,
$$

where $N$ is the total number of trials between each judgment, $A, B, C$, and $D$ represent frequency of each type of trial, and $w_{i}$ represents their weights constrained by the absolute subjective ranking $a>b=c>d$.

By the use of these two equations, the asymmetrical effect of a noncontingent preexposure to a cue and a consequence can be explained, provided that we also assume that these subjective weights of the new evidence can change as a function of the previous experience, as is suggested by the results of Experiment 4. Accordingly, after having detected that there was no contingency between the two events during preexposure, when the subjects were again asked about the relationships between both events in the next block, they gave less weight to the type $a$ trials when estimating the NewEvidence and, hence, the current contingency. Thus, judgment updating should be sensitive to subjective prior experience, and in this way, it is possible to explain the asymmetrical effect of prior noncontingency, as can be seen in the simulation of the model (see Table A1 in the Appendix).

However, the belief revision model cannot be applied in its present form to the cue competition effect when multiple cues are used. In order to extend the model to explain configural learning and multiple-cue effects, it will be necessary to assume new statistical rules to compute the NewEvidence between judgments. But for present purposes and in order to explain the results of Experiment 3 , in which two cues were used, it suffices to assume that, when several cues are presented together, the $\beta$ parameter is reduced when estimating the effect of any one of the single cues. This reduction should produce a delay in the participant's encoding of the accuracy of the cues, as was apparent in this experiment. In any case, the model requires further assumptions to be able to explain phenomena such as blocking, configural learning, and recency effects.

In summary, the asymmetrical effect of a previous noncontingent experience on covariation learning, as well as its influence on changing prior beliefs about the subjective value of each type of trial and other phenomena, such as the frequency of judgment effect (Catena et al., 1998), raises difficulties for current associative models of covariation detection in humans, which might be overcome by the inclusion of a statistical decision rule (Price \& Yates, 1995; Shanks, 1991), which would also account for judgments when information is presented in a summary table.

A number of recent findings that include conditioned inhibition (Allan, 1993; Chapman \& Robbins, 1990), however, strongly suggest the existence of an associative mechanism, and recent reviews (Allan, 1993; Price \& Yates, 1995; Shanks, 1995) have emphasized its explanatory power in accounting for other effects in causal and covariation learning, such as cue competition (Wasserman, 1990; but see Waldman \& Holyoak, 1992) and order and recency effects (López, Shanks, Almaraz, \& Fernández, 1998; but see Waldman \& Holyoak, 1997). Overall results on human covariation learning point to the possibility of a flexible architecture in which the information integration system giving rise to the judgment could rely on the action of a cognitive mechanism or an associative one, or on the two working in conjunction (Catena, Megías, Fresse, \& Maldonado, 1999; Price \& Yates, 1995). But the activation conditions for these mechanisms when humans estimate the covariation between events remain to be determined by future research. 


\section{REFERENCES}

Allan, L. (1993). Human contingency judgment: Rule based or associative? Psychological Bulletin, 114, 435-448.

Alloy, L. B., \& TABACHNIK, N. (1984). Assessment of covariation by humans and animals: The joint influence of prior expectations and current situational information. Psychological Review, 91, 112-149.

Baker, A. G., Berbrier, M., \& Vallée-Tourangeau, F. (1989). Judgements of a $2 \times 2$ contingency table: Sequential processing and the learning curve. Quarterly Journal of Experimental Psychology, 41B, 65-97.

BAKER, A. G., \& MACKINTOSH, N. (1977). Excitatory and inhibitory conditioning following uncorrelated presentations of CS and UCS. Animal Learning \& Behavior, 5, 315-319.

Baker, A. G., \& MacKintosh, N. (1979). Preexposure to CS alone, US alone or CS and US uncorrelated: Latent inhibition, blocking by context or learned irrelevance? Learning \& Motivation, 13, 391-416.

Baker, A. G., Mercier, P., Vallée-Tourangeau, F., Frank, R., \& PAN, M. (1993). Selective associations and causality judgments: The presence of a strong causal factor may reduce judgments of a weaker one. Journal of Experimental Psychology: Learning, Memory, \& Cognition, 19, 414-432.

BENEDICT, J. O. (1991). Judgment of covariation in classical and instrumental conditioning contexts. Bulletin of the Psychonomic Society, 29, 457-459.

Bennet, C. I., Maldonado, A., \& MacKintosh, N. (1995). Learned irrelevance is not the sum of exposure to CS and US. Quarterly Journal of Experimental Psychology, 48B, 117-128.

Bonardi, C., \& HALL, G. (1996). Learned irrelevance: No more than the sum of CS and US preexposure effects? Journal of Experimental Psychology: Animal Behavior \& Processes, 22, 183-191.

Catena, A., Maldonado, A., \& Cándido, A. (1998). The effect of the frequency of judgment and the type of trials on covariation learning. Journal of Experimental Psychology: Human Perception \& Performance, 24, 481-495.

Catena, A., Megías, J. L., Fresse, B., \& Maldonado, A. (1999). Frequency of judgment: Evidence of associative and rule-based cognitive mechanisms. Manuscript submitted for publication.

Chapman, G. B. (1991). Trial order affects cue interaction in contingency judgment. Journal of Experimental Psychology: Learning, Memory, \& Cognition, 17, 837-854.

Chapman, G. B., \& Robbins, S. J. (1990). Cue interaction in human contingency judgment. Memory \& Cognition, 18, 537-545.

CHENG, P. (1997). From covariation to causation: A causal power theory. Psychological Review, 104, 367-405.

Cheng, P., \& Novick, L. (1992). Covariation in natural causal induction. Psychological Review, 99, 365-382.

CroCKer, J. (1982). Biased questions in judgment of covariation studies. Personality \& Social Psychology Bulletin, 8, 214-220.

DICKINSON, A., \& BURKE, J. (1996). Within compound associations mediate the retrospective reevaluation of causality judgments. Quarterly Journal of Experimental Psychology, 49B, 60-80.

EDWARDS, W. (1954). The theory of decision making. Psychological Bulletin, 51, 380-417.

Hallam, S. C., Matzel, L. D., Sloat, J. S., \& Miller, R. R. (1990). Excitation and inhibition as a function of posttraining extinction of the excitatory cue used in Pavlovian inhibition training. Learning \& Motivation, 21, 59-84.

HogaRTH, R. M., \& EINHORN, H. J. (1992). Order effects in belief updating: The belief-adjustment model. Cognitive Psychology, 24, 1-55.

Holland, P., \& RESCORLA, R. (1982). Behavioral studies of associative learning in animals. Annual Review of Psychology, 33, 265-308.

JENKINS, H. J., \& WARD, W. C. (1965). Judgment of contingency between responses and outcomes. Psychological Monographs: General \& Applied, 79 (1, No.594).

KaO, S. F., \& WASSERMAN, E. A. (1993). Assessment of an information integration account of contingency judgment with examination of subjective cell importance and method of information presentation. Journal of Experimental Psychology: Learning, Memory, \& Cognition, 19, 1363-1386.
LEVIN, I., Wasserman, E., \& KaO, S. (1993). Multiple methods for examining biased information use in contingency judgments. Organizational Behavior \& Human Decision Processes, 55, 228-250.

López, F., Shanks, D., Almaraz, J., \& Fernández, P. (1998). Effects of trial order on contingency judgments: A comparison of associative and probabilistic contrast accounts. Journal of Experimental Psychology: Learning, Memory, \& Cognition, 24, 672-694.

MaIER, S., \& SEligman, M. (1976). Learned helplessness: Theory and evidence. Journal of Experimental Psychology: General, 105, 3-46.

Maldonado, A., Catena, A., García, I., \& Cándido, A. (1996) Efecto de asimetria de la no-contingencia previa en el aprendizaje de covariacion [The effect of prior noncontingency asymmetry on learning of covariation]. Paper presented at the VIII Congreso de la Sociedad Española de Psicología Comparada, Malaga.

Maldonado, A., Martos, R., \& Ramírez, E. (1991). Human contingency judgments in terms of previous controllability and actual contingency. Quarterly Journal of Experimental Psychology, 43B, 347-360.

MARKMAN, A. B. (1989). LMS rules and the inverse base-rate effect: Comments on Gluck and Bower (1988). Journal of Experimental Psychology: General, 118, 417-421.

MATUTE, H. (1995). Human reactions to uncontrollable outcomes: Further evidence for superstition rather than helplessness. Quarterly Journal of Experimental Psychology, 48B, 142-157.

Matute, H., ARCediano, F., \& Miller, R. (1996). Test question modulates cue competition between causes and between effects. Journal of Experimental Psychology: Learning, Memory, \& Cognition, 22, 182-196.

Matzel, L. D., Schachtman, R. T., \& Miller, R. (1988). Learned irrelevance exceeds the sum of CS preexposure and US preexposure deficits. Journal of Experimental Psychology: Animal Behavior Processes, 14, 311-319.

MilleR, R., \& MatUTE, H. (1996). Animal analogues of causal judgment. Psychology of Learning \& Motivation, 34, 133-166.

Price, P. C., \& YATES, J. F. (1995). Associative and rule-based accounts of cue interaction in contingency judgment. Journal of Experimental Psychology: Learning, Memory, \& Cognition, 21, 1639-1655.

RESCORLA, R. (1968). Probability of shocks in the presence and absence of CS in fear conditioning. Journal of Comparative \& Physiological Psychology, 66, 1-5.

RESCORLA, R. A., \& WAGNER, A. R. (1972). A theory of Pavlovian conditioning: Variations in the effectiveness of reinforcement and nonreinforcement. In A. H. Black \& W. F. Prokasy (Eds.), Classical conditioning II: Current research and theory (pp. 64-99). New York: Appleton-Century-Crofts.

SHAKLEE, H., \& MIMS, M. (1982). Sources of error in judging event covariations: Effects of memory demands. Journal of Experimental Psychology: Learning, Memory, \& Cognition, 8, 208-224.

SHANKs, D. R. (1985). Continuous monitoring of human contingency judgment across trials. Memory \& Cognition, 13, 158-167.

SHANKS, D. R. (1987). Acquisition functions in contingency judgment. Learning \& Motivation, 18, 147-166.

SHANKS, D. R. (1991). On similarities between causal judgments in experienced and described situations. Psychological Science, 2, 341-350.

SHANKS, D. [R.] (1995). Is human learning rational? Quarterly Journal of Experimental Psychology, 48A, 257-279.

SHANKS, D. R., \& Dickinson, A. (1987). Associative accounts of causality judgment. In G. H. Bower (Ed.), The psychology of learning and motivation (Vol. 21, pp. 229-261). San Diego: Academic Press.

Shanks, D. R., LÓPEZ, F., Darby, R., \& Dickinson, A. (1996). Distinguishing associative and probabilistic contrast theories of human contingency judgment. In D. [R.] Shanks, K. [J.] Holyoak, \& D. L. Medin (Eds.), The psychology of learning and motivation: Causal learning (Vol. 34, pp. 265-310). San Diego: Academic Press.

Shanks, D. R., Pearson, S. M., \& Dickinson, A. (1989). Temporal contiguity and the judgment of causality by human subjects. Quarterly Journal of Experimental Psychology, 41 B, 139-159.

TAssonI, C. J. (1995). The least mean squares network with information coding: A model of cue learning. Journal of Experimental Psychology Learning, Memory, \& Cognition, 21, 193-204.

Van Hamme, L., \& Wasserman, E. A. (1994). Cue competition in 
causality judgments: The role of nonpresentation of compound stimulus elements. Learning \& Motivation, 25, 127-151.

WaldmanN, M. [R.], \& Holyoak, K. [J.] (1992). Predictive and diagnostic learning within causal models: Asymmetries in cue competition. Journal of Experimental Psychology: General, 121, 222-236.

WALDMANN, M. R., \& HolYOAK, K. J. (1997). Determining whether causal order affects cue selection in human contingency learning: Comments on Shanks and López (1996). Memory \& Cognition, 25, 125-134.

WASSERMAN, E. A. (1990). Attribution of causality to common and distinctive elements of compound stimuli. Psychological Science, 1, 298-302.

Wasserman, E. A., Dorner, W. W., \& KaO, S. F. (1990). Contributions of specific cell information to judgments of interevent contingency. Journal of Experimental Psychology: Learning, Memory, \& Cognition, 16, 509-521.

Williams, D. (1996). A comparative analysis of negative contingency learning in humans and nonhumans. In D. [R.] Shanks, K. [J.] Holyoak, \& D. L. Medin (Eds.). The psychology of learning and motivation. Causal learning (Vol. 34, pp. 89-131). San Diego: Academic Press.

\section{APPENDIX}

Here we present the equations and the parameters we used to simulate our model. Trial sequences were the same as those used in Experiment 1. The results obtained with these parameters and the equations below are presented in Table Al.

The model of Catena et al. (1998) is based on anchoring and belief revision assumptions (Hogarth \& Einhorn, 1992), to explain response mode and sequential learning effects. As was explained in the General Discussion section, the first equation of the model has three terms: a parameter adjustment rate $(\beta)$, NewEvidence since the last judgment, and the judgment $\left(J_{i}\right)$, where $i$ indicates trial number:

$$
\left.J_{i}=J_{i-1}+\beta \text { (NewEvidence }-J_{i-1}\right) .
$$

Initial belief values were set to 0 . The value for $\beta$ was fixed at 0.7 ( $\beta$ is assumed not to change as a function of experience). NewEvidence is assumed to be computed on the information presented from the last judgment, $J_{i-1}$, to the current judgment, $J_{i}$. There are many ways to perform this computation. We have assumed a weighted $\Delta D$ rule, which allows the computation even when information is reduced to that of a single trial:

$$
\text { NewEvidence }=\left(w_{1} \cdot a+w_{2} \cdot b+w_{3} \cdot c+w_{4} \cdot d\right) / N \text {. }
$$

Weight parameters were derived from the G0 group of Experiment $4 \mathrm{~B}$, as this group estimated the value of each type of
Table A1

Simulated Cumulative Covariation Judgment Based on

Catena, Maldonado, and Cándido's (1998) Model, Applied to Experiment 1, With the Parameters Derived From the G0 Group of Experiment 4B

\begin{tabular}{lccccrrrrr}
\hline & \multicolumn{4}{c}{ Phase 1 } & & \multicolumn{4}{c}{ Phase 2 } \\
\cline { 8 - 10 } \cline { 7 - 9 } Group & B1 & B2 & B3 & B4 & & B1 & B2 & B3 & B4 \\
\hline G+5 & & & & & & 28 & 36 & 39 & 40 \\
G0+5 & 7 & 9 & 10 & 10 & & 16 & 18 & 19 & 19 \\
G-5 & & & & & -14 & -18 & -19 & -20 \\
G0-5 & 7 & 9 & 10 & 10 & -21 & -30 & -33 & -34 \\
\hline
\end{tabular}

Note- $-\mathrm{G}$ stands for group. The numbers in each group name represents the contingency $(+5=.5 ;-5=-.5 ; 0=$ noncontingency), as is explained in Table 1.

trial before and after a noncontingent experience. The mean estimations on the influence of each type of trial (see Table 3) were converted into a scale ranged from +1 to -1 , taking into account the between-subjects variability and the previous assumptions of the model (Catena et al., 1998).

Consequently, to estimate the weight of each cell, we assigned the value 1 to the larger estimation of the G0 subjects (i.e., 4.1, maximum $x$, which was the value of the type $a$ trial before training; see Table 3). Hence, the rule to estimate the weight in each cell was

$$
w=p x /(\operatorname{maximum} x)
$$

where maximum $x$ was the larger value, $x$ indicates the value assigned to each type of trial by the G0 subjects (see Table 3 ), and $p$ is a weighting factor depending on the variability in each type of trial. Finally, according to the assumptions of the model (Catena et al., 1988), we also assumed that the weights for the $b$ and $c$ trial types were the same, and we used the mean of both values to calculate the parameters. Hence, the initial parameters before any training were $w_{1}=1, w_{2}=-.5, w_{3}=-.5$, and $w_{4}=$ .4 , whereas after the noncontingent preexposure, the parameter values changed to $w_{1}=.6, w_{2}=-.6, w_{3}=-.6$, and $w_{4}=.3$.

(Manuscript received July 13, 1998; revision accepted for publication December 23, 1998.) 